\title{
IL-25 induces airways angiogenesis and expression of multiple angiogenic factors in a murine asthma model
}

\author{
Xiujuan Yao ${ }^{1}$, Wei Wang ${ }^{2 *}$, Yan Li ${ }^{3}$, Ping Huang ${ }^{2}$, Qian Zhang ${ }^{1}$, Jingjing Wang ${ }^{4}$, Wen Wang ${ }^{3}$, Zhe Lv², Yunqing An²,
} Jianguo Qin ${ }^{5}$, Chris J Corrigan ${ }^{6}$, Kewu Huang ${ }^{3}$, Yongchang Sun ${ }^{1}$ and Sun Ying ${ }^{2,6}$

\begin{abstract}
Background: Th2-promoting cytokine IL-25 might contribute to bronchial mucosal vascular remodelling in asthma through its receptor expressed by vascular endothelial and vascular smooth muscle cells.

Methods: By utilising a newly established chronic asthma murine model induced by direct exposure of the airways to IL-25 alone, we examined effects of IL-25 on angiogenesis, vascular remodelling and expression of angiogenic factors, compared changes with those in a "classical" ovalbumin (OVA)-induced murine asthma model. IL-25 and OVA were intranasally instilled into the airways of BALB/C mice for up to 55 days. Airways vessels and angiogenic factors, including Von Willebrand Factor (VWF), amphiregulin, angiogenin, endothelin-1, transcription factor ERG, basic fibroblast growth factor (bFGF), epidermal growth factor (EGF), insulin-like growth factor (IGF-1) and vascular endothelial growth factor (VEGF) in lung sections, homogenates and BAL fluid were detected and quantified by immunostaining or enzyme linked immunosorbent assay (ELISA). An in house assay was also utilised to compare the effects of IL-25 and other Th2-cytokines on angiogenesis by human vascular endothelial cells.

Results: Repetitive intranasal challenge with IL-25 alone or OVA alone in OVA-presensitised animals significantly increased peribronchial VWF ${ }^{+}$vessels in the murine airways, which was associated with remarkably elevated expression of amphiregulin, angiogenin, endothelin-1, bFGF, EGF, IGF-1, VEGF and ERG. IL-25, but not Th-2-cytokines induced human angiogenesis in vitro.
\end{abstract}

Conclusions: The data suggest that chronic exposure of murine airways to IL-25 alone is able to reproduce a local angiogenic milieu. Thus, blocking IL-25 may attenuate vascular remodelling and improve outcomes in asthma patients.

Keywords: IL-25, IL-25 receptor, Asthma, Pathogenesis, Angiogenesis, Fibrosis, Murine model

\section{Introduction}

Asthma is a chronic inflammatory disorder of the airways which is associated with airways hyperresponsiveness that leads to recurrent episodes of wheezing and breathlessness [1]. The pathophysiology of asthma has been intensively investigated. Nowadays, it is well accepted that in addition to inflammation, airways remodelling is also a hallmark feature of asthma which has been implicated in regulating disease severity and control [2-4]. Airways remodelling

\footnotetext{
*Correspondence: wy_robin@ccmu.edu.cn

${ }^{2}$ Department of Immunology, School of Basic Medical Sciences, Capital Medical University, Beijing, People's Republic of China

Full list of author information is available at the end of the article
}

refers to structural changes including loss of epithelial integrity, thickening of the subepithelial basement membrane caused by fibrosis, goblet cell metaplasia and submucosal gland enlargement, increased airway smooth muscle mass, increased airways vascularity and vascular remodelling [2-4]. Although the notion that airways remodelling is one of the key features of asthma is well established, the underlying mechanisms have not been fully clarified. It is now hypothesised that inflammation and vascular remodelling in asthma are partially interdependent, reflecting a dysregulated wound healing process resulting in extracellular matrix deposition and angiogenesis [5].

Angiogenesis of bronchial mucosal vessels may be observed in response to a wide variety of stimuli, including 
chronic airways inflammation. It may be defined as either the formation of new vessels by sprouting from preexisting vessels and/or lengthening and enlargement of existing vessels. Angiogenesis is a complex multiphase process, potentially involving a great number of growth factors, cytokines, chemokines, enzymes and other factors $[4,6]$. These are produced by resident stromal or inflammatory cells, creating a "vascular remodelling milieu". Notwithstanding that vascular endothelial growth factor (VEGF) is considered to be a key mediator in this process, the specific roles of other molecules remain to be defined [6].

IL-25 (IL-17E), a distant member of the IL-17 family has been demonstrated to induce Th2-type immune responses, indicating that it may contribute to the pathogenesis of asthma and thereby this "vascular remodelling milieu" [7]. In mice, overexpression or exogenous administration of IL-25 produces an asthma-like inflammatory response, including increased serum IgE production, blood eosinophilia and lung eosinophilic infiltrates, epithelial cell hyperplasia/hypertrophy, increased mucus secretion and airways hyperreactivity [8-11]. Conversely, blockade of IL-25 remarkably reduced Th2 cytokine production and airways inflammation [7,12], and in addition ameliorated airways remodelling changes including peribronchial collagen deposition and smooth muscle hyperplasia, with corresponding reduction of hyperreactivity [12].

Our recent previous data further underline the ability of IL-25 to effect airways inflammation and fibrosis in vivo. Firstly, we detected elevated expression of IL-25 and its receptor in the human asthmatic bronchial mucosa at baseline and following allergen challenge [13]. Secondly, we showed that chronic intranasal instillation of IL-25 into murine airways is able to induce acute and chronic inflammatory responses reminiscent of human asthma [14]. Thirdly, we showed that IL-25, by binding to its IL-25R/IL-17RB, but not its IL-17RA receptor enhanced human lung vascular endothelial cell proliferation and angiogenesis in vitro by increasing the expression by these cells of basic fibroblast growth factor (bFGF) and VEGF and VEGF receptors [15,16].

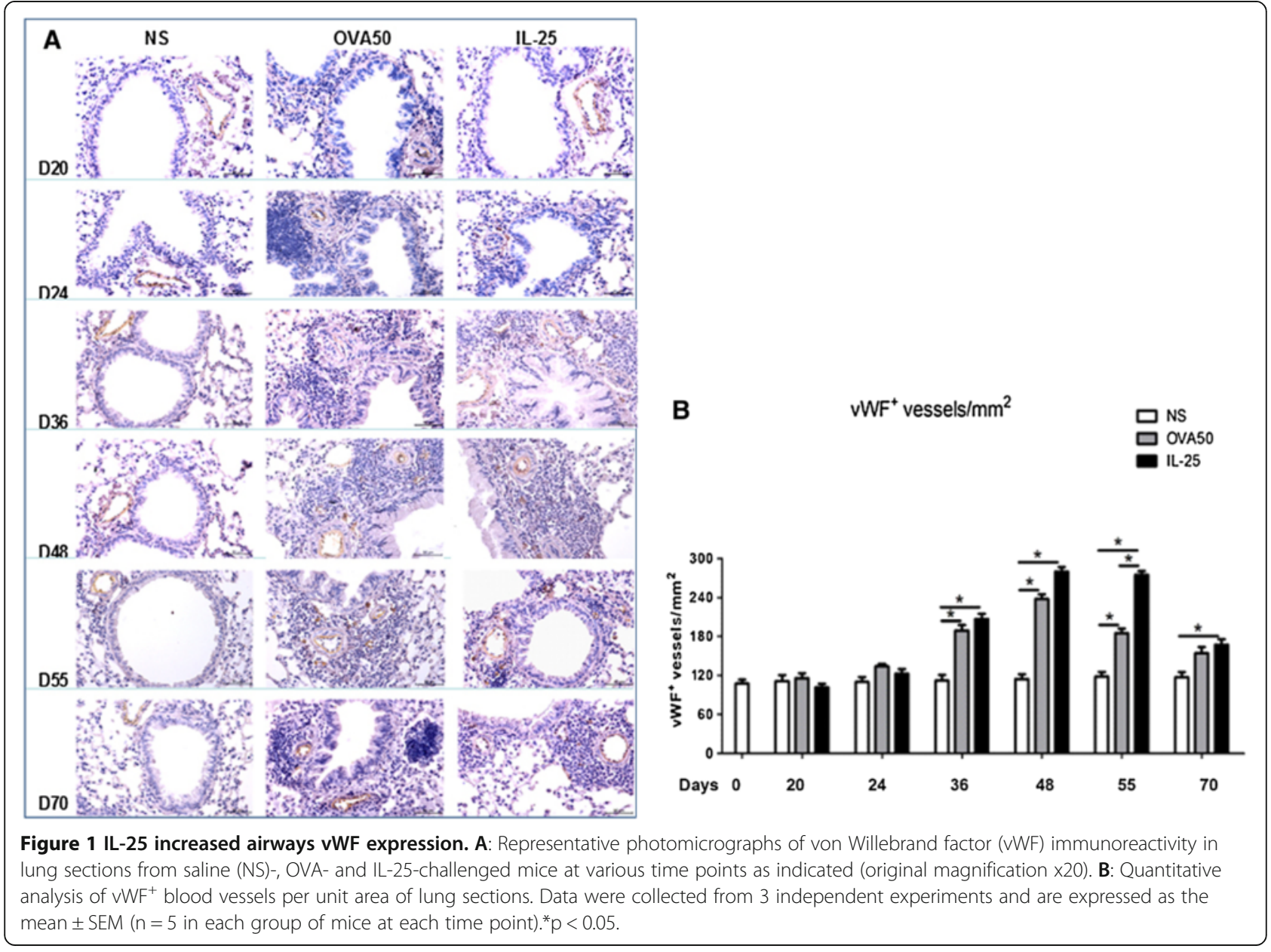


Nevertheless, there is still a paucity of information as to the full range of potential effects of IL-25 in promoting a "vascular remodelling milieu" in the setting of asthmatic inflammation in vivo. In particular, angiogenic and growth factors such as epidermal growth factor (EGF), ETS-related gene (ERG), insulin-like growth factor 1 (IGF-1), amphiregulin, angiogenin and endothelin-1, together with transforming growth factor- $\beta 1$ (TGF- $\beta 1$ ), VEGF and bFGF have been suggested to have potential to participate in this "remodelling milieu"[4].

Consequently we set out to extend our previous studies to examine the direct effects of IL-25 alone on the production of these mediators in the airways in vivo in our murine model of chronic asthma. In this model, we compared in parallel the effects of direct intranasal instillation of IL-25 with those of intranasal OVA administered to mice pre-sensitised to mount an IgE response to OVA by intraperitoneal sensitization (a "classical" murine asthma model). Finally, we compared the direct effects of IL-25 with those of IL-4, IL-5 and IL-13 in promoting angiogenesis in a human in vitro model.

\section{Methods}

Animals

Female BALB/c mice (8-10 wk old) were purchased from Vital River Laboratories (Beijing, China) and maintained in a pathogen free mouse facility located in the Department of Laboratory Animal Sciences, Capital Medical University, Beijing, China. They were kept in $12 \mathrm{~h}$ light and $12 \mathrm{~h}$ dark with free access to food and water. All experiments were approved by the Institutional Animal Care and Use Committee (IACUC).

\section{Allergen- and IL-25 intranasal instillation protocol}

Mice were divided into 3 groups, including a group serially challenged with OVA (a "classical" allergen-induced asthma model) $[14,17]$, a group challenged with saline as a negative control and a group serially challenged with IL-25. Briefly, OVA-challenged group were first sensitized by intraperitoneal injection of OVA (SigmaAldrich, Beijing, China, $100 \mu \mathrm{g}$ emulsionised in $\mathrm{Al}[\mathrm{OH}]$ $3 /$ dose) on days 0 and 12 . Then $50 \mu \mathrm{g}$ of OVA (OVA50) in $50 \mu \mathrm{L}$ saline/dose were further administered to these

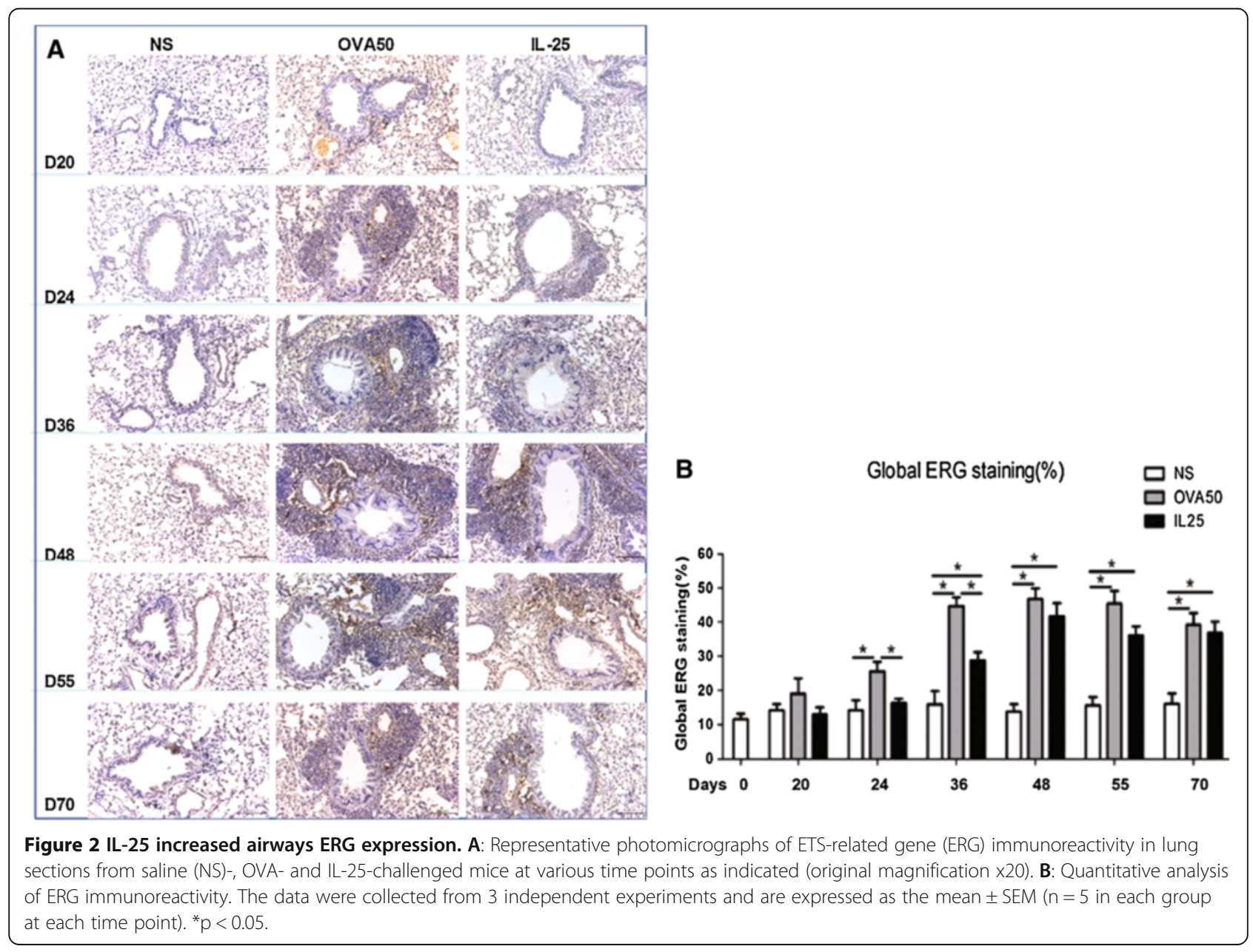


mice daily by nasal instillation from days 18 to 23 . IL-25 challenged group were not sensitized to OVA on days 0 and 12 , but suffered daily nasal instillation with recombinant mouse IL-25 (mIL-25, R\&D Systems, $2 \mu \mathrm{g}$ in 50 $\mathrm{uL}$ saline) from days 18 to 23 [14,18]. Subsequently, these mice were further challenged intranasally either with OVA (OVA50 group) or with IL-25 (IL-25 group) every 2 days for a further 30 days. 5 mice in each group were observed for a further 17 days after stopping the challenges. Saline negative group was intraperitoneally injected with the same amount of $\mathrm{Al}[\mathrm{OH}] 3$ on days 0 and 12 , then nasally administered with saline at time points corresponding to those in the other groups.

\section{Bronchoalveolar lavage fluid collection and preparation of lung tissue homogenates}

Bronchoalveolar lavage fluid (BALF) was collected from mice immediately following euthanasia as previously described [14]. Supernatants were stored at $-80^{\circ} \mathrm{C}$ until used. Right lung tissue $(100 \mathrm{mg})$ was homogenized in $2 \mathrm{~mL}$ PBS containing $1 \%$ Triton-X100 and a protease inhibitor cocktail tablet (Roche Diagnostics $\mathrm{GmbH}$, Mannheim, Germany). Debris was removed by centrifugation and the supernatants collected and stored at $-80^{\circ} \mathrm{C}$ until used for measurement of analytes.

\section{Analysis of VEGF and bFGF}

VEGF and bFGF concentrations in BALF and lung homogenates were measured using commercial ELISA kits (eBioscience, San Diego, CA). In brief, $100 \mu$ l of capture antibody were added to each plate well overnight at $4^{\circ} \mathrm{C}$. After washing 5 times, $100 \mu \mathrm{l}$ of acidified BALF or lung homogenate were added to the plate wells which were then incubated for $2 \mathrm{~h}$ at room temperature (RT). After 5 washes, $100 \mu \mathrm{l}$ of detection antibody were added to each well and the plates incubated for a further $1 \mathrm{hr}$ at $37^{\circ} \mathrm{C}$. After washing, $100 \mu \mathrm{l}$ of avidin-HRP labelled antibody were added and the plates incubated for $30 \mathrm{~min}$ at RT. Plates were washed again 5 times and $100 \mu \mathrm{l}$ of substrate solution added to each well for incubation at RT in the dark. After colour development, $50 \mu \mathrm{l}$ of stop solution were added to each well. Absorbance was measured at $450 \mathrm{~nm}$ using a GloMAc-Multi Detection System (e7801, Shanghai Promega Biological Products, Ltd, China). Concentrations of VEGF and bFGF in BALF and lung homogenates were determined by interpolation from the standard curve.

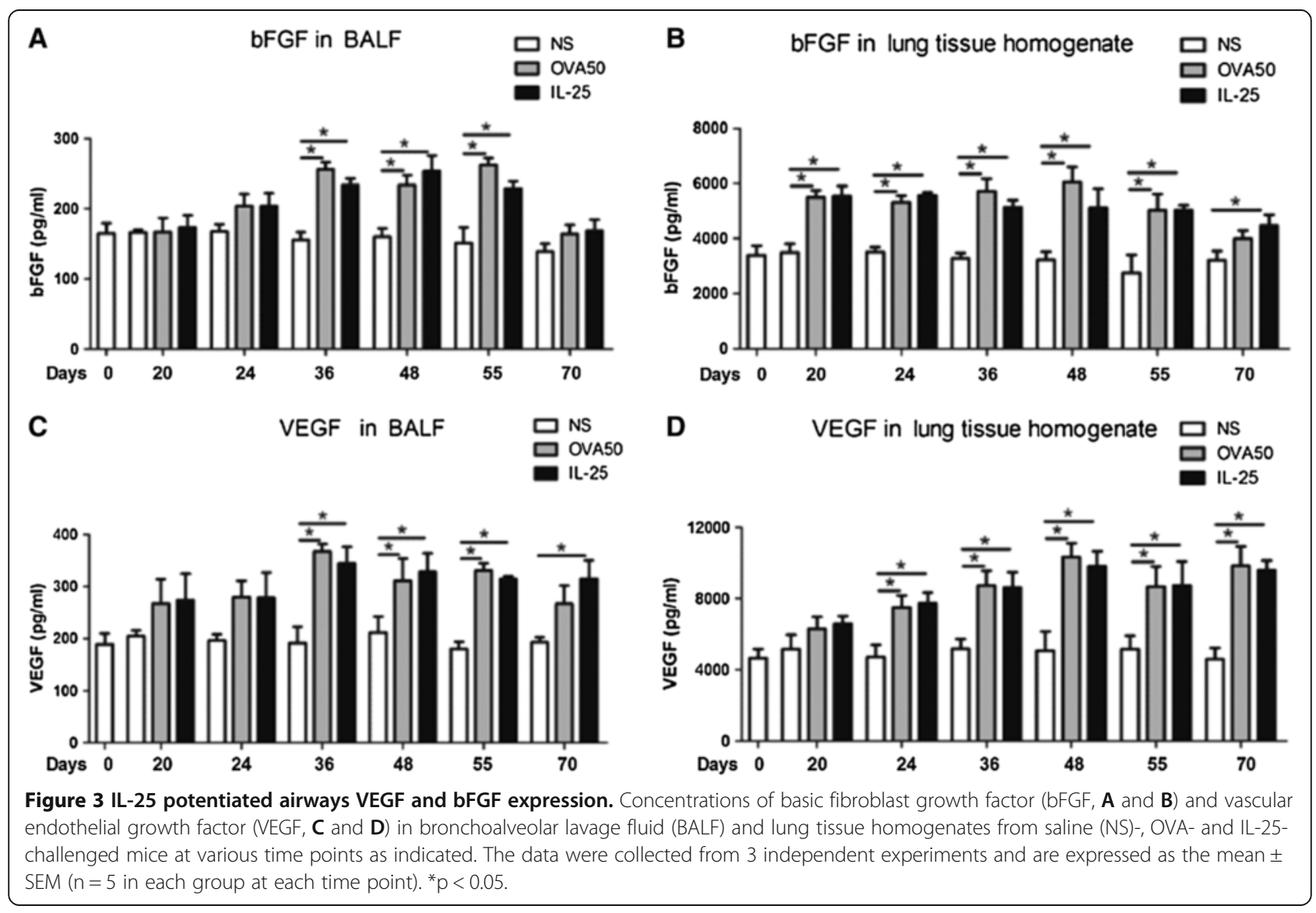




\section{Lung immunohistochemistry}

Immunohistochemistry was used to detect bronchial vascular biomarkers. The primary monoclonal antibodies against mouse transcription factor (ERG, 1:80), epidermal growth factor (EGF, 1:100), insulin-like growth factor (IGF-1, 1:400), endothelin-1 (1:600), angiogenin (1:400) and amphiregulin (1:500) were purchased from Abcam (Hong Kong, China). The primary monoclonal antibody against mouse Von Willebrand factor (vWF, 1:50) was purchased from CHEMICON International, Inc. MA 01730. The PAP (peroxidase anti-peroxidase) technique was used as previously described [13-15]. Positively staining cells were detected using the glucose oxidase-DAB-nickel method [19]. At least 8 random high-power fields $(200 \times$ total magnification $)$ of each mice in the 3 groups should be independently analysed by 2 observers in a blinded fashion on a Leica DM6000B microscope (Leica, Wetzlar, Germany) connected with a Leica Application Suite Version 3.6. The data were expressed as percentages of positive stained areas per unit area of entire lung sections.

\section{In vitro angiogenesis assay}

It is believed that Th2-type cytokines such as IL-4, IL-5 and IL-13 play a significant role in airways remodelling in asthma. To further investigate whether these cytokines also have effects on angiogenesis, we used a well established in vitro angiogenesis assay (AngioKit; TCS CellWorks, Buckingham, UK) $[15,16]$, which is based on co-culture of HUVEC over a monolayer of irradiated fibroblasts. Briefly, cultures were incubated at $37^{\circ} \mathrm{C}$ in a humidified atmosphere with $5 \% \mathrm{CO}_{2}$. Culture medium with recombinant human IL-4, IL-5, IL-13 and IL-25 (10 ng/ml, R\&D Systems, Abingdon, UK) [15,16] was replenished on days 4,7 , and 9. Culture medium alone and recombinant human VEGF-A (R\&D Systems, $10 \mathrm{ng} / \mathrm{ml}$ ) $[15,16]$ served as negative and positive assay controls, respectively. On day 11 cultured cells were fixed and vascular structures visualized by labelling with mouse anti-CD31 according to the manufacturer's instructions. Multiple photomicrographs $(\times 4$ objective) (4 separate fields per well) were taken at clock points 12, 3, 6 and 9, and angiogenesis in each field of view quantified using

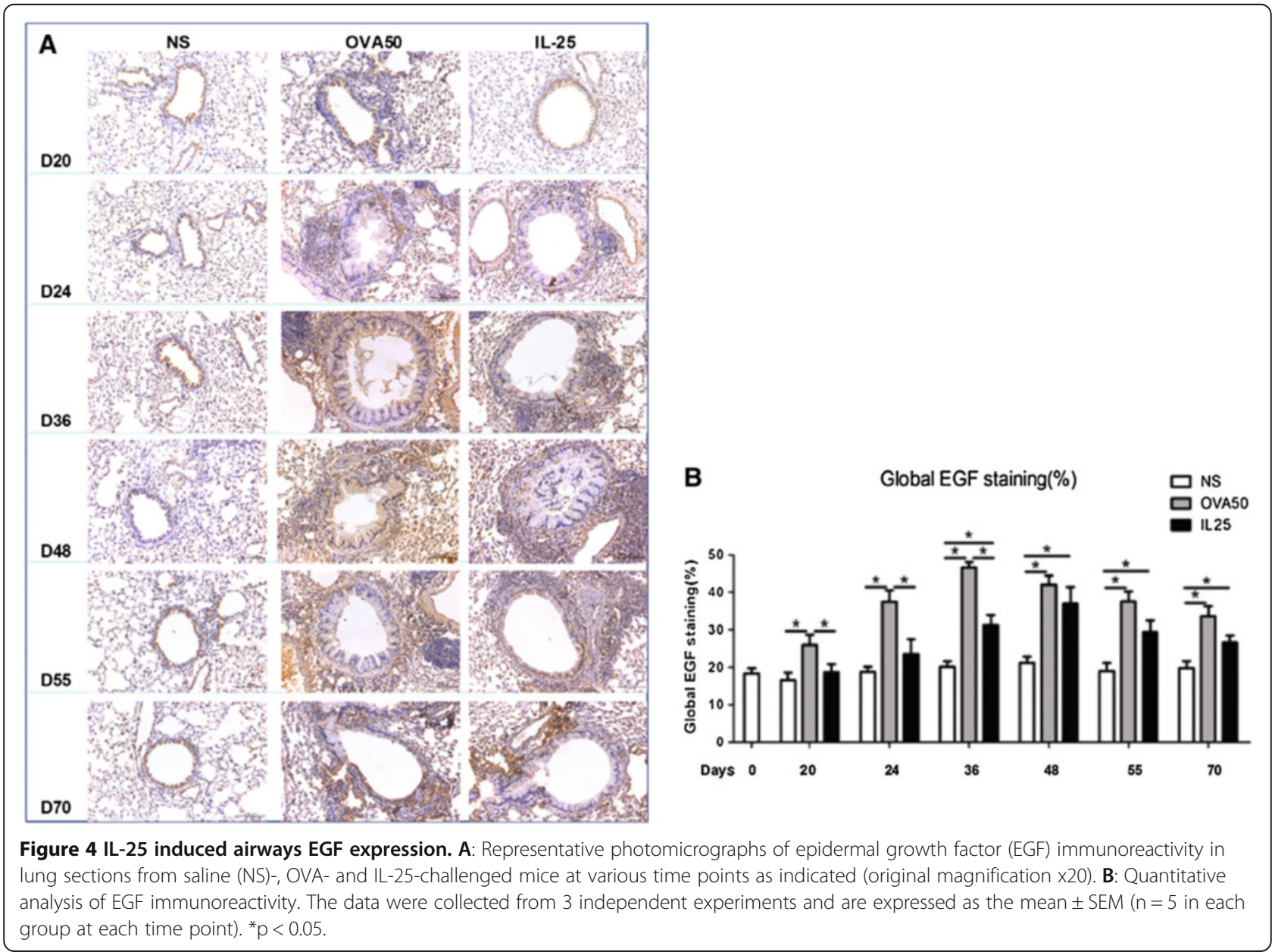


image analysis software (AngioSys; TCS CellWorks) as described previously $[15,16]$. The analysis software segmented the images using a grey level threshold tool to select CD31labelled cells. The resultant binary images were skeletonized and branch points removed to determine the total lengths of individual tubules. Branch points were counted and the total area of CD31 labelling determined from the original binary images, permitting overall numbers of vascular junctions, tubules, and tubule length to be determined.

\section{Statistical analysis}

The results were analyzed using InStat 2.01 software (GraphPad, San Diego, CA, USA). Differences between experimental groups were analyzed using 2-way parametric ANOVA followed by a Bonferroni post test or the Student's unpaired $t$ test. Data are presented as the mean \pm SEM. For all tests, $\mathrm{p}$ values less than 0.05 were considered significant.

\section{Results}

\section{IL-25 increased airways vascularity}

Immunoanalysis showed that IL-25, as compared with saline challenge induced marked airways angiogenesis as indicated by significantly elevated numbers of peribronchial, $\mathrm{vWF}^{+}$immunoreactive blood vessels from day 36 and persisting until the end of the experiment (day 70) (Figure 1). Similarly, OVA challenged animals also showed elevated $\mathrm{vWF}^{+}$immunoreactive vessels from days 36 to 55 which however declined by day 70 (Figure 1). Correspondingly, IL-25 challenge also significantly increased the expression of global airways ERG immununoreactivity from day 36 until day 70 (Figure 2). OVA challenge similarly increased ERG expression although this was evident slightly earlier from day 24 until the end of the experiment (Figure 2).

\section{IL-25 potentiated airways parenchymal and luminal VEGF and bFGF expression}

IL-25, as compared with saline challenge of the murine airways induced significantly elevated mean concentrations of bFGF, as measured by ELISA, in lung homogenates from day 20 and in BALF from day 36, with resolution in BALF by day 70 (Figure 3). Mean VEGF concentrations were also significantly elevated in lung homogenates from day 24 and BALF from day 36 until

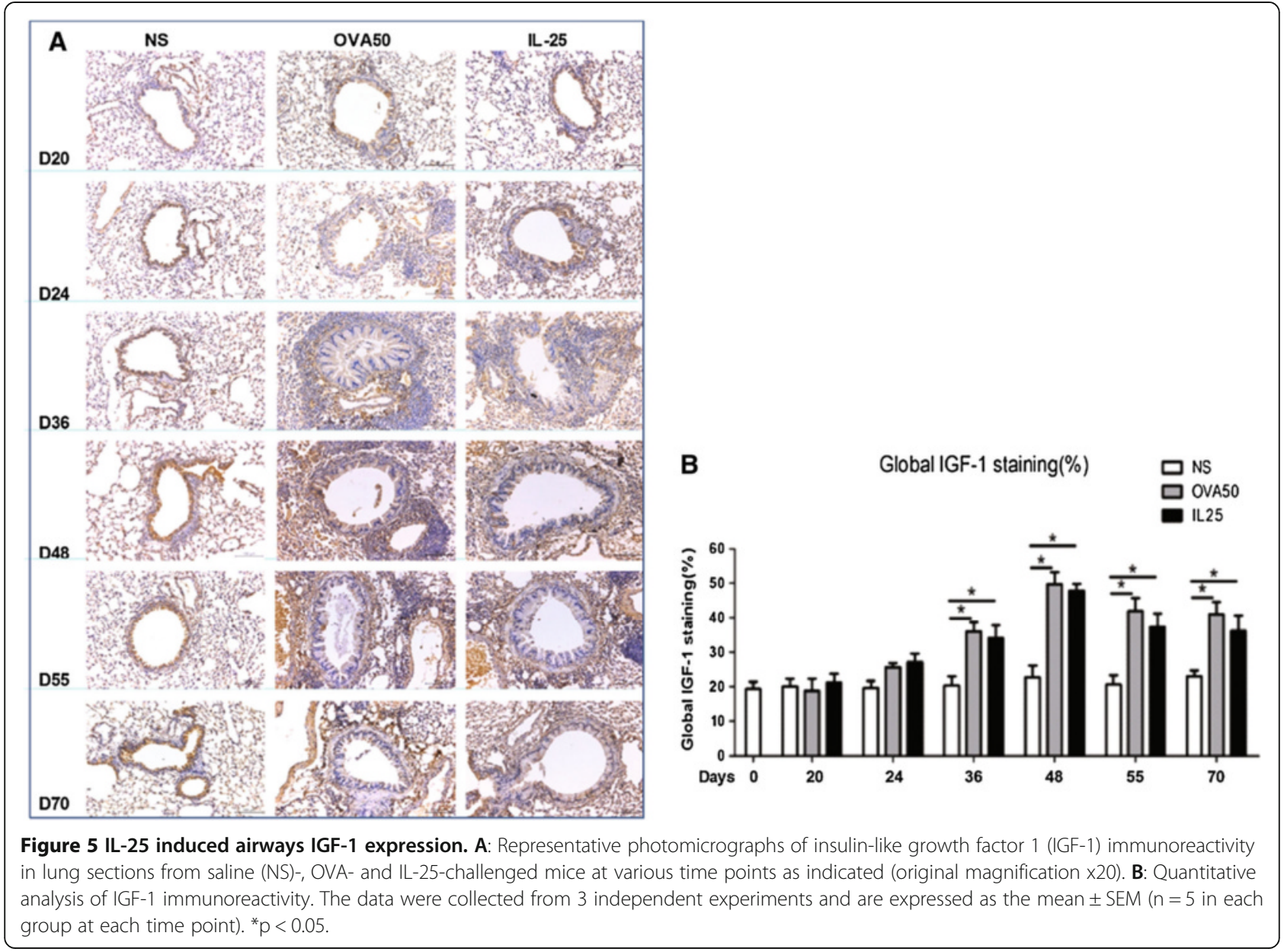


the end of the experiment. OVA challenge induced similar changes in bFGF and VEGF concentrations in both compartments with a similar time course (Figure 3).

\section{IL-25 induced airways EGF and IGF-1 expression}

Immunoanalysis showed that IL-25, as compared with saline challenge of the airways significantly increased the expression of lung and peribronchial EGF and IGF-1 immunoreactivity from day 36 until the end of the experiment (Figures 4 and 5). OVA challenge produced a similar effect which was apparent earlier (from day 20) and more marked between days 20 and 36 in the case of EGF (Figures 4 and 5).

\section{IL-25 induced elevated airways endothelin-1, angiogenin and amphiregulin expression}

Immunoanalysis showed that IL-25 as compared with saline challenge of the airways significantly increased expression of lung and peribronchial endothelin-1, angiogenin and amphiregulin immunoreactivity from day 24 (endothelin-1) or day 36 until the end of the experiment. OVA challenge induced similar but more marked changes which were apparent earlier (From days 20 or 24) (Figures 6, 7 and 8).

\section{IL-25, but not IL-4, IL-5 or IL-13 induced angiogenesis in vitro}

In an in vitro angiogenesis assay, IL-25, but not IL-4, IL-5 or IL-13, induced lengthening and branching of microvascular tubules by HUVEC as compared with VEGF, which was used as a positive control (Figure 9).

\section{Discussion}

Accumulating reports have firmly established angiogenesis and vascular remodelling as features of asthma pathophysiology which could have an important impact on day to day symptomatology as well as the natural history of the disease [20-22]. So far, relatively little is known about the mechanisms of these changes. Here we demonstrate that chronic challenge of murine airways with IL-25 alone has the capacity to induce angiogenesis in vivo and increase local production of a variety of

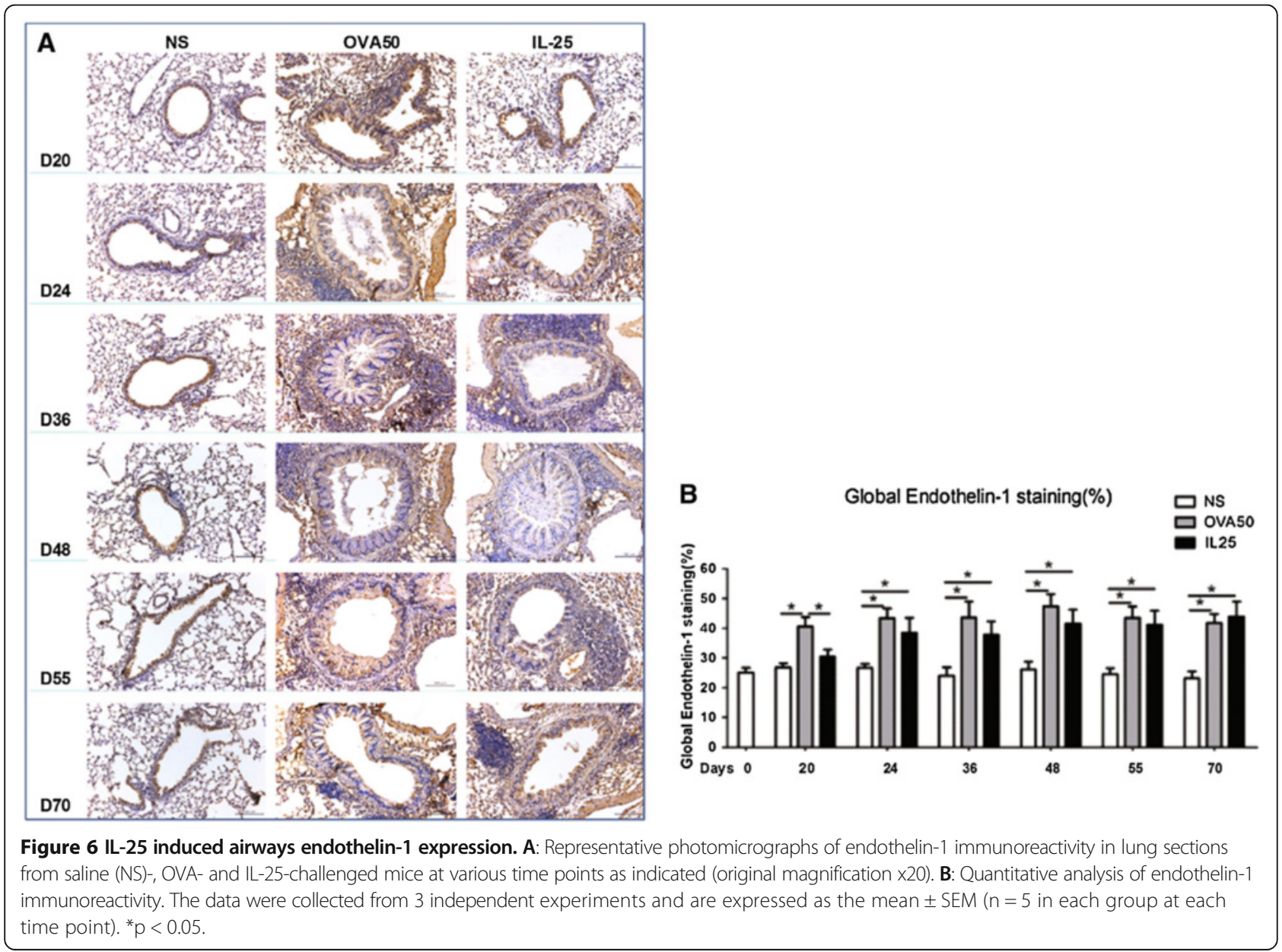



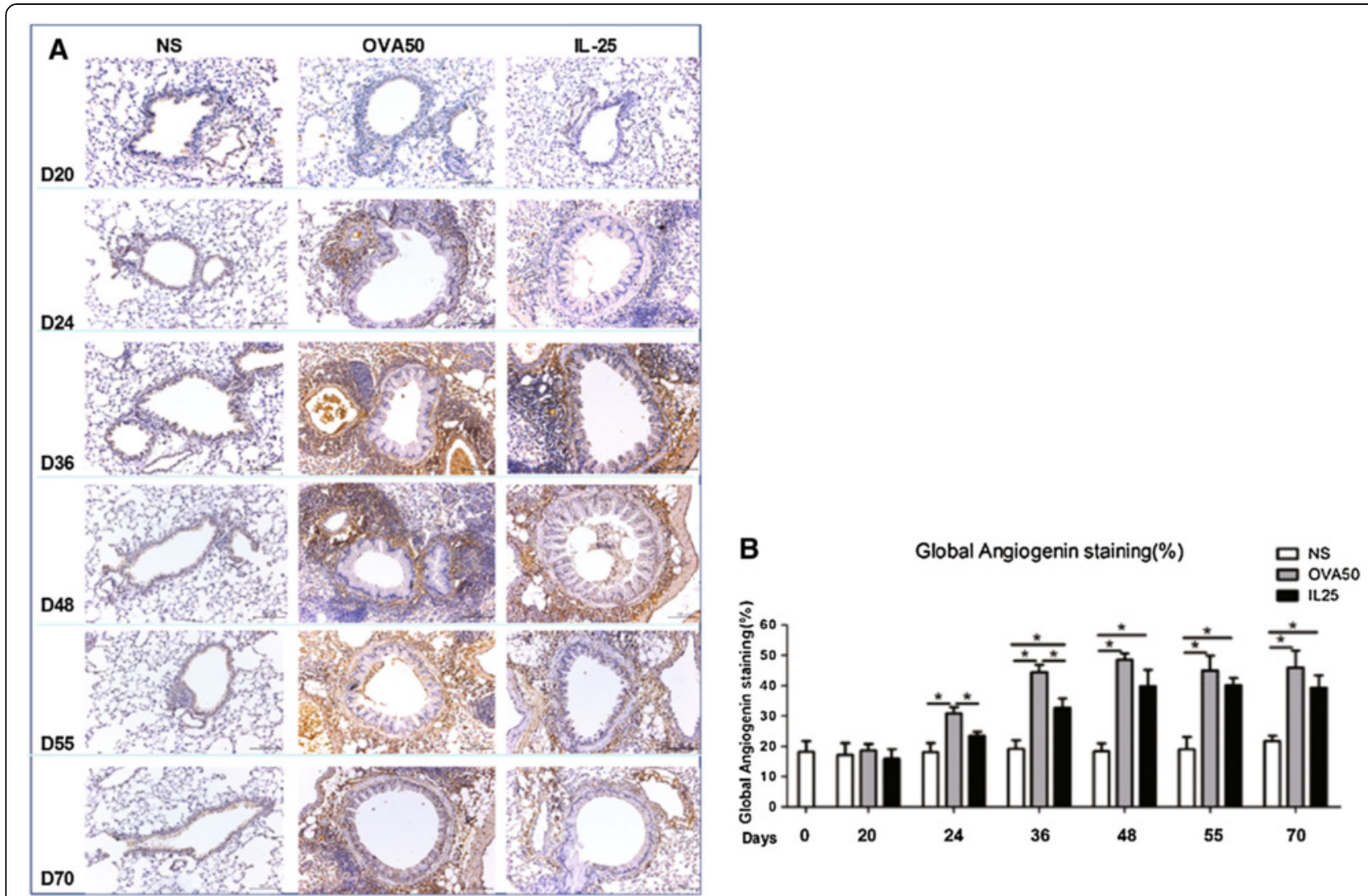

Figure 7 IL-25 induced airways angiogenin expression. A: Representative photomicrographs of angiogenin immunoreactivity in lung sections from saline (NS)-, OVA- and IL-25-challenged mice at various time points as indicated (original magnification $\times 20$ ). B: Quantitative analysis of angiogenin immunoreactivity. The data were collected from 3 independent experiments and are expressed as the mean \pm SEM $(n=5$ in each group at each time point). ${ }^{*} p<0.05$.

angiogenic mediators in a setting of chronic inflammation resembling that induced by "classical" murine asthma models which require prior IgE sensitization of the animals. The only difference was that some of the changes were more marked and some occurred slightly earlier with OVA, as compared with IL-25 challenge, perhaps at least partly reflecting the release of mast cell mediators in the airways. This does not of course imply that the molecular mechanisms inducing these changes are necessarily similar, but does underline the potential pleiotropic activities of IL-25 alone. Further, we have shown that, although a variety of Th2 cytokines as well as IL-25 have been implicated in causing remodelling changes in human and murine airways in asthma, only IL-25, and not IL-4, IL-5 or IL-13 is able directly to induce angiogenesis by human vascular endothelial cells. This extends our previous data [16] and is also congruent with previous study [12] showing that angiogenesis is increased in the airways of mice sensitized to, then challenged with house dust mite and that this can be abrogated by IL-25 blockade, possibly at least partly by reduced recruitment of CXCR2 endothelial cell progenitors from the bone marrow.

Vascular endothelial growth factor (VEGF) is currently considered to be a key angiogenic factor in asthma [23], while the expression of bFGF is also upregulated in human asthma and has been correlated with the increased vascularity of the bronchial mucosa and lung function $[24,25]$. Our data showed that both IL-25 and OVAchallenged mice showed increased production of both mediators, first in the lung parenchyma and in the airways lumen, with a time course reflecting that of elevated blood vessel signature markers including vWF and ERG. vWF is a common marker for evaluating angiogenesis in vivo and ex vivo [12], while ERG is a member of the ETS (erythroblast transformation-specific) family of transcription factors which is selectively expressed by endothelial cells, specific haematopoetic cells and pre-cartilage cells. It has been shown that ERG plays an important role in blood vessel homeostasis and angiogenesis by regulating a variety of endothelial cellular functions including survival, junctional stability and migration [26]. 


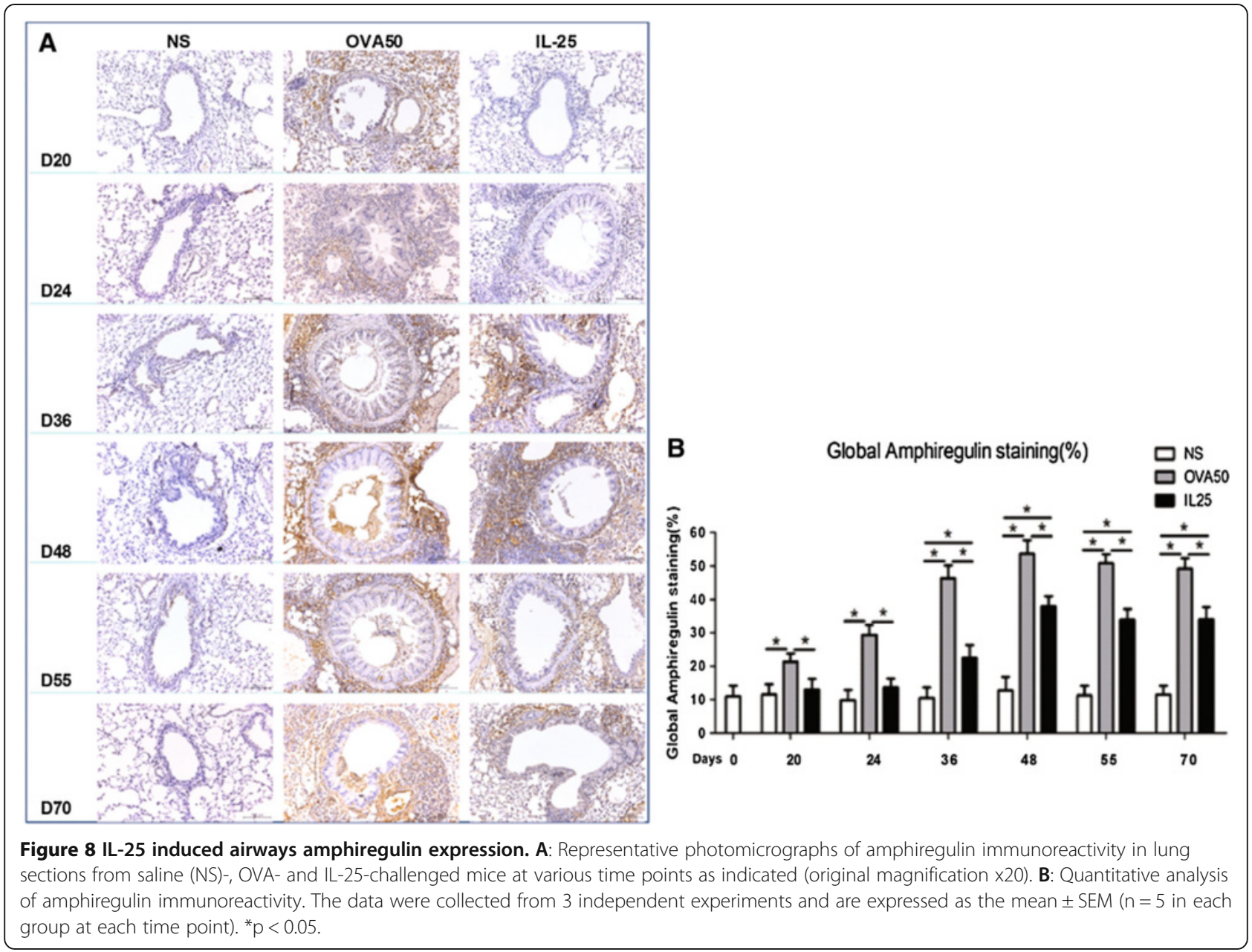

Physiologically, airways blood vessel homeostasis likely reflects a balance between the production of endogenous angiogenic activators and inhibitors. When the balance is perturbed because of over-production of angiogenic factors and/or down-regulation of inhibitors, a proangiogenic niche ensues and aberrant vascularisation occurs [2-4]. Aside from VEGF and FGF, which are heparin binding growth factors, several other proangiogenic mediators have been implicated in regulating neovascularisation in airways. Circulating concentrations of insulin-like growth factor (IGF-I) have been linked with airways vascular remodelling in patients with chronic airways disease [27]. Inhibition of epidermal growth factor (EGF) receptor signalling ameliorated airways hyperreactivity and remodelling in a murine model of chronic asthma [28]. Amphiregulin, a member of the EGF family and another ligand of the EGF receptor, has been shown to promote proliferation of airways epithelial and smooth muscle cells and mucin gene expression [29]. Neutralization of amphiregulin also prevented cultured endothelial cells from forming tubes [30]. In addition to growth factors, other pro-angiogenic mediators such as endothelin-1 and angiogenin also contribute to aberrant intrapulmonary neovascularisation and remodelling [31-33]. Endothelin-1, in addition to promoting synthesis of ECM proteins by epithelial cells and fibroblasts, myofibroblast differentiation and proliferation of mesenchymal cells, also induces migration and proliferation of vascular cells [34]. Angiogenin, first isolated from conditioned medium of colonic carcinoma cell cultures, is a potent tumour-derived angiogenic factor but also plays a role in several non-malignant vasculoproliferative disorders, and like VEGF induces vascular endothelial cell proliferation, migration and tubule formation [35]. Our data show that all of these pro-angiogenic agents have the potential to be upregulated by IL- 25 alone in the setting of asthma-like inflammation of the airways in vivo, with a time course congruent with that of angiogenesis.

\section{Conclusions}

Our data do not distinguish between direct and indirect effects of IL-25 in causing angiogenesis, although we 


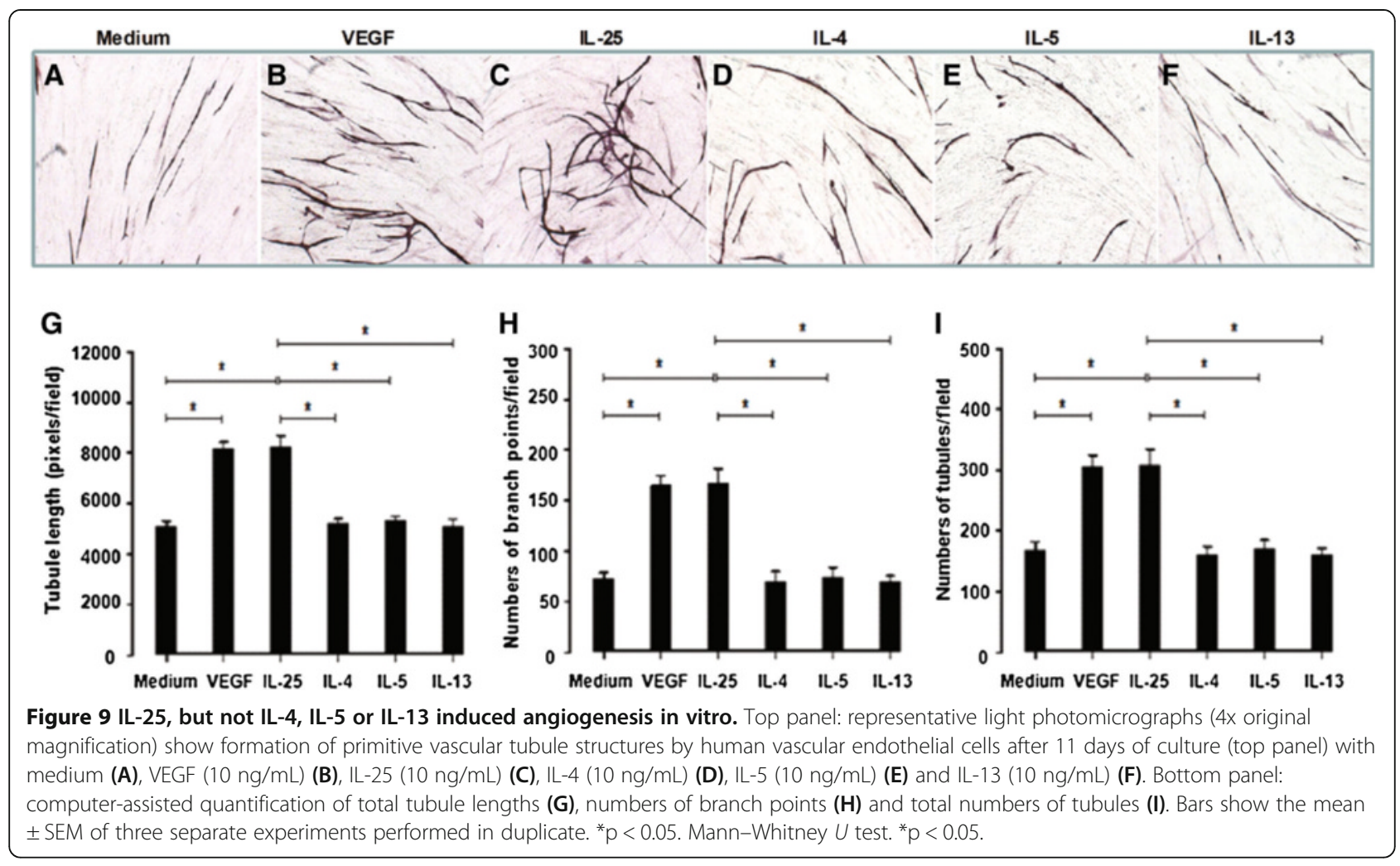

have previously shown $[15,16]$ that IL-25 acts directly on endothelial cells to induce angiogenesis, albeit at least partly through increasing VEGF and bFGF expression by these cells, whereas other Th2-type cytokines (IL-4, IL-5, IL-13), as shown in this study, do not. Angiogenesis likely involves many cell types including fibroblasts and vascular smooth muscle cells [2-6]. We have further preliminary data suggesting that IL-25 also induces expression of VEGF and EGF fibrotic factors by human lung fibroblasts (in preparation). We conclude that IL-25 may be a master switch for the production of many important pro-angiogenic mediators by a diverse variety of airways structural cells. Targeting IL-25 may provide a novel therapeutic approach to altering the natural history of asthmatic inflammation and associated symptoms.

\section{Abbreviations}

AHR: Airways hyperresponsiveness; bFGF: Basic fibroblast growth factor; ECM: Extracellular matrix; EGF: Epidermal growth factor; ERG: ETS-related gene ETS, erythroblast transformation- specific; HUVEC: Human umbilical vein endothelial cells; IGF-1: Insulin-like growth factor 1; IHC: Immunohistochemistry; IL-25: Interleukin 25: IL-25R: Interleukin-25 receptor: OVA: Ovalbumin; VEGF: Vascular endothelial growth factor; vWF: Von Willebrand factor.

\section{Competing interests}

The authors declare that they have no competing interests.

\section{Authors' contributions}

$\mathrm{XY}$ designed and made the experiments, analyzed the data, drafted and revised the manuscript. YL, PH, QZ, JW, WW, ZL, YA and JQ participated in the experiments and analysis of the data. CC and YS contributed to the critical revision of the article for intellectual content. WW, KH and SY contributed to the conception and design of the study, the analysis and interpretation of the data, revision of the article and final approval of the version to be published. All authors read and approved the final manuscript.

\section{Acknowledgements}

We gratefully acknowledge financial support from the National Natural Science Foundation of China (81102250, 81170039, 81373177, 81471594), the Science and Technology Project of the Beijing Municipal Education Commission (KM201110025005), the PhD Programs Foundation of the Ministry of Education of China (20091107110006,20101107110003), Key Projects in the National Science \& Technology Pillar Program during the Twelfth Five-year Plan Period (2012BAl05B02), AstraZeneca China Respiratory Research Awards 2011

\section{Author details}

'Department of Respiratory Medicine, Beijing Tongren Hospital, Capital Medical University, Beijing, People's Republic of China. ${ }^{2}$ Department of Immunology, School of Basic Medical Sciences, Capital Medical University, Beijing, People's Republic of China. ${ }^{3}$ Department of Respiratory and Critical Care Medicine, Beijing Chao-Yang Hospital, Capital Medical University \& Beijing Institute of Respiratory Medicine, Beijing, People's Republic of China. ${ }^{4}$ Department of Laboratory Animal Sciences, Capital Medical University, Beijing, People's Republic of China. ${ }^{5}$ Dongfang Hospital, The Second Clinical Medical College of Beijing University of Chinese Medicine, Beijing, People's Republic of China. ${ }^{6}$ King's College London, MRC \& Asthma UK Centre in Allergic Mechanisms of Asthma, Division of Asthma, Allergy \& Lung Biology, London, UK. 
Received: 2 December 2014 Accepted: 27 February 2015

Published online: 18 March 2015

\section{References}

1. Bateman ED, Hurd SS, Barnes PJ, Bousquet J, Drazen JM, FitzGerald M, et al. Global strategy for asthma management and prevention: GINA executive summary. Eur Respir J. 2008;31(1):143-78.

2. Bergeron C, Tulic MK, Hamid Q. Airway remodelling in asthma: from benchside to clinical practice. Can Respir J. 2010;17(4):e85-93.

3. Manuyakorn W, Howarth PH, Holgate ST. Airway remodelling in asthma and novel therapy. Asian Pac J Allergy Immunol. 2013;31(1):3-10.

4. Alagappan VK, de Boer WI, Misra VK, Mooi WJ, Sharma HS. Angiogenesis and vascular remodeling in chronic airway diseases. Cell Biochem Biophys. 2013;67(2):219-34.

5. Kudo M, Ishigatsubo Y, Aoki I. Pathology of asthma. Front Microbiol. 2013;4:263.

6. Zanini A, Chetta A, Imperatori AS, Spanevello A, Olivieri D. The role of the bronchial microvasculature in the airway remodelling in asthma and COPD. Respir Res. 2010;11:132

7. Angkasekwinai P, Park H, Wang YH, Chang SH, Corry DB, Liu YJ, et al. Interleukin 25 promotes the initiation of proallergic type 2 responses. J Exp Med. 2007;204(7):1509-17.

8. Hurst SD, Muchamuel T, Gorman DM, Gilbert JM, Clifford T, Kwan S, et al. New IL-17 family members promote Th1 or Th2 responses in the lung: in vivo function of the novel cytokine IL-25. J Immunol. 2002;169(1):443-53.

9. Fort MM, Cheung J, Yen D, Li J, Zurawski SM, Lo S, et al. IL-25 induces IL-4, IL-5, and IL-13 and Th2-associated pathologies in vivo. Immunity. 2001;15(6):985-95.

10. Pan G, French D, Mao W, Maruoka M, Risser P, Lee J, et al. Forced expression of murine IL-17E induces growth retardation, jaundice, a Th2-biased response, and multiorgan inflammation in mice. J Immunol. 2001;167(11):6559-67.

11. Kim MR, Manoukian R, Yeh R, Silbiger SM, Danilenko DM, Scully S, et al. Transgenic overexpression of human IL-17E results in eosinophilia, B-lymphocyte hyperplasia, and altered antibody production. Blood. 2002;100(7):2330-40.

12. Gregory LG, Jones CP, Walker SA, Sawant D, Gowers KH, Campbell GA, et al. IL-25 drives remodelling in allergic airways disease induced by house dust mite. Thorax. 2013;68(1):82-90.

13. Corrigan CJ, Wang W, Meng Q, Fang C, Eid G, Caballero MR, et al. Allergeninduced expression of IL-25 and IL-25 receptor in atopic asthmatic airways and late-phase cutaneous responses. J Allergy Clin Immunol. 2011;128(1):116-24.

14. Yao XJ, Huang KW, Li Y, Zhang Q, Wang JJ, Wang W, et al. Direct comparison of the dynamics of IL-25- and 'allergen'-induced airways inflammation, remodelling and hypersensitivity in a murine asthma model. Clin Exp Allergy. 2014;44(5):765-77.

15. Corrigan CJ, Wang W, Meng Q, Fang C, Wu H, Reay V, et al. T-helper cell type 2 (Th2) memory T cell-potentiating cytokine IL-25 has the potential to promote angiogenesis in asthma. Proc Natl Acad Sci U S A. 2011;108(4):1579-84.

16. Wang W, Fan YQ, Lv Z, Yao XJ, Huang KW, Meng Q, et al. Interleukin-25 promotes basic fibroblast growth factor expression by human endothelial cells through interaction with IL-17RB, but not IL-17RA. Clin Exp Allergy. 2012;42(11):1604-14.

17. McMillan SJ, Lloyd CM. Prolonged allergen challenge in mice leads to persistent airway remodelling. Clin Exp Allergy. 2004;34(3):497-507.

18. Riesenfeld E, Allen GB, Bates JH, Poynter ME, Wu M, Aimiand S, et al. The temporal evolution of airways hyperresponsiveness and inflammation. J Allergy Ther. 2012;1(5):1-7.

19. Wang C, Shu SY, Guo Z, Cai YF, Bao X, Zeng C, et al. Immunohistochemical localization of mu opioid receptor in the marginal division with comparison to patches in the neostriatum of the rat brain. J Biomed Sci. 2011;18:34.

20. Chetta A, Zanini A, Foresi A, Del Donno M, Castagnaro A, D'Ippolito R, et al. Vascular component of airway remodeling in asthma is reduced by high dose of fluticasone. Am J Respir Crit Care Med. 2003;167(5):751-7.

21. Li X, Wilson JW. Increased vascularity of the bronchial mucosa in mild asthma. Am J Respir Crit Care Med. 1997;156(1):229-33.

22. Salvato G. Quantitative and morphological analysis of the vascular bed in bronchial biopsy specimens from asthmatic and non-asthmatic subjects. Thorax. 2001;56(12):902-6.

23. Walters EH, Soltani A, Reid DW, Ward C. Vascular remodelling in asthma. Curr Opin Allergy Clin Immunol. 2008;8(1):39-43.

24. Hoshino M, Takahashi M, Aoike N. Expression of vascular endothelial growth factor, basic fibroblast growth factor, and angiogenin immunoreactivity in asthmatic airways and its relationship to angiogenesis. J Allergy Clin Immunol. 2001;107(2):295-301.

25. Bissonnette EY, Madore AM, Chakir J, Laviolette M, Boulet LP, Hamid Q, et al. Fibroblast growth factor-2 is a sputum remodeling biomarker of severe asthma. J Asthma. 2014;51(2):119-26.

26. Birdsey GM, Dryden NH, Shah AV, Hannah R, Hall MD, Haskard DO, et al. The transcription factor Erg regulates expression of histone deacetylase 6 and multiple pathways involved in endothelial cell migration and angiogenesis. Blood. 2012;119(3):894-903.

27. Elsasser TH. Insulin-like growth factor-l: a traffic control device on the road to tissue recovery. Am J Physiol Regul Integr Comp Physiol. 2003;285(4):R722-3.

28. Le Cras TD, Acciani TH, Mushaben EM, Kramer EL, Pastura PA, Hardie WD, et al. Epithelial EGF receptor signaling mediates airway hyperreactivity and remodeling in a mouse model of chronic asthma. Am J Physiol Lung Cell Mol Physiol. 2011:300(3):L414-21.

29. Berasain C, Avila MA. Amphiregulin. Semin Cell Dev Biol. 2014;28:31-41.

30. Busser B, Sancey L, Brambilla E, Coll $J$, Hurbin A. The multiple roles of amphiregulin in human cancer. Biochim Biophys Acta. 2011;1816(2):119-31.

31. Gregory LG, Jones CP, Mathie SA, Pegorier S, Lloyd CM. Endothelin-1 directs airway remodeling and hyper-reactivity in a murine asthma model. Allergy. 2013;68(12):1579-88.

32. Simcock DE, Kanabar V, Clarke GW, Mahn K, Karner C, O'Connor BJ, et al. Induction of angiogenesis by airway smooth muscle from patients with asthma. Am J Respir Crit Care Med. 2008;178(5):460-8.

33. Enomoto Y, Orihara K, Takamasu T, Matsuda A, Gon Y, Saito H, et al. Tissue remodeling induced by hypersecreted epidermal growth factor and amphiregulin in the airway after an acute asthma attack. J Allergy Clin Immunol. 2009;124(5):913-20. e911-917.

34. Shao D, Park JE, Wort SJ. The role of endothelin-1 in the pathogenesis of pulmonary arterial hypertension. Pharmacol Res. 2011;63(6):504-11.

35. Kishimoto K, Liu S, Tsuji T, Olson KA, Hu GF. Endogenous angiogenin in endothelial cells is a general requirement for cell proliferation and angiogenesis. Oncogene. 2005;24(3):445-56.

\section{Submit your next manuscript to BioMed Central and take full advantage of:}

- Convenient online submission

- Thorough peer review

- No space constraints or color figure charges

- Immediate publication on acceptance

- Inclusion in PubMed, CAS, Scopus and Google Scholar

- Research which is freely available for redistribution 